\title{
DE-STAR: Phased-array laser technology for planetary defense and other scientific purposes
}

Gary Hughes, Philip Lubin, J. Bible, Jesse Bublitz, Josh Arriola, et al.

Gary B. Hughes, Philip Lubin, J. J. Bible, Jesse Bublitz, Josh Arriola, Caio Motta, Jon Suen, Isabella Johansson, Jordan Riley, Nilou Sarvian, Jane Wu, Andrew Milich, Mitch Oleson, Mark Pryor, "DE-STAR: Phased-array laser technology for planetary defense and other scientific purposes," Proc. SPIE 8876, Nanophotonics and Macrophotonics for Space Environments VII, 88760J (24 September 2013); doi: 10.1117/12.2026401

Event: SPIE Optical Engineering + Applications, 2013, San Diego, California, United States 


\title{
DE-STAR: Phased-Array Laser Technology for Planetary Defense and Other Scientific Purposes
}

\author{
Gary B. Hughes ${ }^{\mathrm{a}}$, Philip Lubin ${ }^{\mathrm{b}}$, J. J. Bible ${ }^{\mathrm{b}}$, Jesse Bublitz ${ }^{\mathrm{b}}$, Josh Arriola ${ }^{\mathrm{b}}$, Caio \\ Motta $^{\mathrm{b}}$, Jon Suen ${ }^{\mathrm{b}}$, Isabella Johansson ${ }^{\mathrm{b}}$, Jordan Riley ${ }^{\mathrm{b}}$, Nilou Sarvian ${ }^{\mathrm{b}}$, Jane Wu ${ }^{\mathrm{b}}$, \\ Andrew Milich $^{\mathrm{b}}$, Mitch Oleson ${ }^{\mathrm{b}}$ and Mark Pryor ${ }^{\mathrm{c}}$ \\ ${ }^{a}$ Statistics Department, California Polytechnic State University, San Luis Obispo, CA 93407-0405, \\ gbhughes@calpoly.edu \\ ${ }^{\mathrm{b}}$ Physics Department, University of California, Santa Barbara, CA 93106-9530 \\ ${ }^{c}$ Vorticy Inc, San Diego, CA 92121
}

\begin{abstract}
Current strategies for diverting threatening asteroids require dedicated operations for every individual object. We propose a stand-off, Earth-orbiting system capable of vaporizing the surface of asteroids as a futuristic but feasible approach to impact risk mitigation. We call the system DE-STAR (Directed Energy System for Targeting of Asteroids and exploRation). DE-STAR is a modular phased array of laser amplifiers, powered by solar photovoltaic panels. Lowcost development of test systems is possible with existing technology. Larger arrays could be tested in sub-orbital demonstrations, leading eventually to an orbiting system. Design requirements are established by seeking to vaporize the surface of an asteroid, with ejected material creating a reaction force to alter the asteroid's orbit. A proposed system goal would be to raise the surface spot temperature to $>3,000 \mathrm{~K}$, evaporating all known substances. Engagement distance required for successful diversion depends on the asteroid's mass, composition and approach velocity. Distance to focus and desired surface spot temperature then determine laser array size. Volatile-laden objects (such as comets) $\sim 100 \mathrm{~m}$ wide and approaching at $5 \mathrm{~km} / \mathrm{s}$ could be diverted by initiating engagement at $\sim 0.05 \mathrm{AU}$, requiring a laser array of $\sim 100 \mathrm{~m}$ side length. Phased array configuration allows multiple beams, so a single DE-STAR of sufficient size would be capable of targeting several threats simultaneously. An orbiting DE-STAR could serve diverse scientific objectives, such as propulsion of kinetic asteroid interceptors or other interplanetary spacecraft. Vaporization of debris in Earth orbit could be accomplished with a $\sim 10 \mathrm{~m}$ array. Beyond the primary task of Earth defense, numerous functions are envisioned.
\end{abstract}

Keywords: Asteroid Impact, Directed Energy, Laser Phased Array, Planetary Defense

View presentation video on SPIE's Digital Library: http://dx.doi.org/10.1117/12.2026401

\section{INTRODUCTION}

The Apollo-class asteroid 2012 DA14 was discovered on 23 February 2012 by the Observatorio Astronómico de La Sagra, Granada, Spain [observers Sanchez et al., 2012]. Subsequent observations and calculations revealed that the $\sim 45 \mathrm{~m}$ wide bolide would pass close to Earth the following year. At approximately 19:24 UTC on 15 February 2013, the asteroid approached to within $27,743 \mathrm{~km}$ of Earth's surface, inside the orbit of geosynchronous satellites. The close approach of DA14 was preceded 16 hours by the impact of an unrelated and previously unknown $\sim 14 \mathrm{~m}$ wide Apolloclass asteroid near Chelyabinsk, Russia at approximately 3:20 UTC on the same date. The impact culminated in an aerial explosion and surface impact, creating a shock wave that injured approximately 1,200 people and caused an estimated US\$33 million in property damage. These coinciding events delivered an unambiguous reminder that the risk of Earth impact by asteroids and comets is substantial, from both known and unknown near-Earth orbiting objects.

Current efforts to address the threat of Earth impact include surveillance, orbit determination with impact risk assessment, civil planning, and contemplation of missions to confront objects that might be determined to have a high probability of impacting Earth. Ground-based optical observatories like the Observatorio Astronómico de La Sagra are continually searching for undiscovered asteroids. Orbiting observatories have also made significant contributions, utilizing infrared sensors to detect objects that are difficult to see optically. Observations from many stations around the globe are collected in databases dedicated to cataloging asteroids and comets. Multiple observations of an object's position through time allow determination of orbital characteristics. Propagating a measured orbit into the future provides a mechanism to quantify the risk of Earth impact. Automated orbit determination routines have been developed that exploit information in expanding databases. In addition to searching for and tracking near-Earth asteroids, scientific teams are studying civil response strategies for impacts of varying sizes [Cockell, 2005]. 
Receiving perhaps the most visibility among efforts to confront the threat of an asteroid impact are proposed strategies for diverting an asteroid that is on a collision course with Earth. Diverse methods for orbit alteration have been devised, including nuclear-armed kinetic impactors where the crash and blast would seek to alter the asteroid's orbit and avoid a collision with Earth. In this paper, we offer a novel idea for diverting an asteroid, based on an orbiting directed energy system that would be capable of evaporating the surface of distant asteroids. We call the system DESTAR, for Directed Energy System for Targeting of Asteroids and exploration. We begin by presenting an overview of the asteroid impact threat, focusing on actual scenarios where current orbit deflection tactics may be insufficient. We present a short review of existing impact mitigation strategies. We introduce DE-STAR as a futuristic but feasible approach to impact risk mitigation. We compare and contrast DE-STAR's capabilities with existing schemes for asteroid orbit deflection. We also explore system requirements for a functional DE-STAR, and conclude with a steppeddevelopment roadmap for achieving a DE-STAR system capable of virtually complete planetary defense. Such a system could also be useful for other scientific purposes, which are briefly mentioned in this paper (and discussed in greater detail in other SPIE Optics \& Photonics 2013 Proceedings papers, including [Lubin et al., 2013] and [Bible et al., 2013]).

\section{NATURE OF THE ASTEROID IMPACT THREAT}

\subsection{The Asteroid Population}

There are currently more than 600,000 known asteroids and comets. Most of the objects are Main Belt Asteroids that orbit the Sun between Mars and Jupiter. Main belt asteroids pose no immediate threat of impacting Earth. There are also more than 10,000 known objects with orbits that cross or approach the orbit of Earth, known as NearEarth Asteroids (NEAs). Any object whose orbit crosses Earth's orbit has some potential for collision. The diagram in Fig. 1 depicts the distribution of all known asteroid orbits in the context of the solar system, captured from a video created by Scott Manley at Armagh Observatory [Manley, 2012].

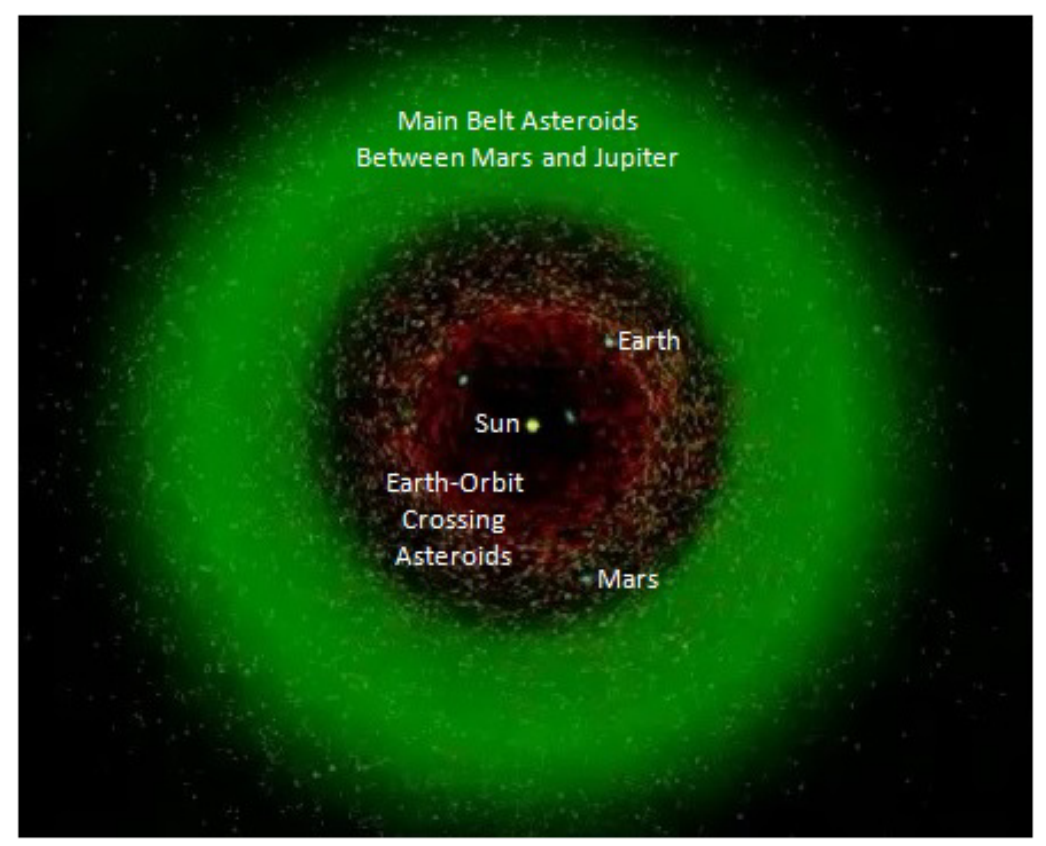

Figure 1. Orbits of known asteroids in the context of the solar system. Main belt asteroids orbit the Sun between Mars and Jupiter, and pose no immediate threat to Earth. A significant number of known asteroids have orbits that approach or cross Earth's orbit, and could potentially impact Earth. Most of the asteroids in threatening orbits have yet to be discovered. 


\subsection{Surveillance and Impact Threat Assessment}

Numerous observatories are dedicated to searching for transient events, including passing asteroids and comets. Integrated surveillance systems are operational, including the Catalina Sky Survey [Larson et al., 2006], NEAT [Helin et al., 1997], LINEAR [Stokes et al., 2000], Pan-STARRS [Kaiser, et al., 2002] and others. Surveillance by ground-based observatories is hindered by atmosphere and solar glare. Orbiting surveillance systems have also been deployed; NASA's Wide-field Infrared Survey Explorer (WISE) spacecraft discovered more than 34,000 asteroids in the main belt between Mars and Jupiter, and 135 near-Earth orbiting objects during its primary mission from January 2010 to February 2011. NASA recently announced the re-activation of WISE for another stint of asteroid hunting. Future missions are also being developed, including Canada's NEOSSat [Wallace et al., 2004], and even by privately funded efforts such as the Sentinel Mission of the B612 Foundation (http://b612foundation.org/).

The total number of asteroids known at the current time represents a fraction of the total asteroid population. Although the discovery rate has increased dramatically with the advent of digital photography and orbiting infrared observatories, much work remains. Larger asteroids are easier to discover, since they are typically bright enough to be seen with ground-based optical observatories. The largest known asteroid is Ceres, with an estimated mean diameter of $952 \mathrm{~km}$; for comparison, Texas is $1,244 \mathrm{~km}$ wide. Unforeseen circumstances notwithstanding, surveillance efforts have likely discovered $100 \%$ of asteroids greater than $500 \mathrm{~km}$ wide, of which four are known, and all were discovered prior to the year 1850. None of the known asteroids $>500 \mathrm{~km}$ wide pose a threat of impacting the Earth in the foreseeable future. Smaller asteroids are much more difficult to find than larger ones. There are an estimated 25 million asteroids that are larger than $100 \mathrm{~m}$, most of which are main belt asteroids. Table 1 lists the estimated cumulative number of asteroids (in all orbits) that are larger than selected asteroid widths.

Table 1. The estimated cumulative number of asteroids in all orbits, including the main belt, that are larger than selected widths.

\begin{tabular}{|c|c|c|c|c|c|c|c|c|c|}
\hline Asteroid Diameter & $100 \mathrm{~m}$ & $500 \mathrm{~m}$ & $1 \mathrm{~km}$ & $5 \mathrm{~km}$ & $10 \mathrm{~km}$ & $50 \mathrm{~km}$ & $100 \mathrm{~km}$ & $500 \mathrm{~km}$ & $1000 \mathrm{~km}$ \\
\hline Cumulative Number & $>25,000,000$ & $2,000,000$ & 750,000 & 90,000 & 10,000 & 600 & 200 & 4 & 0 \\
\hline
\end{tabular}

More relevant to this paper is the estimated distribution of NEAs, which are defined to be asteroids with perihelia less than 1.3AU, and aphelia greater 0.983AU [Stuart, 2001]. An estimated cumulative distribution for NEAs is shown in Fig. 2, based on [Stuart and Binzel, 2004] who conclude that there are 1,090 180 NEAs that are $\geq 1 \mathrm{~km}$ wide.



Figure 2. An estimated cumulative frequency distribution for Near-Earth Asteroids (NEAs) greater than a given diameter, based on [Stuart and Binzel, 2004]. Surveillance efforts have likely discovered over $90 \%$ of NEAs that are $\geq 1 \mathrm{~km}$ wide, but less than $5 \%$ of NEAs that are $\geq 100 \mathrm{~m}$ wide. 
If the estimated distribution of [Stuart and Binzel, 2004] is accurate, then surveillance efforts have discovered $>90 \%$ of NEAs that are $\geq 1 \mathrm{~km}$ wide. The curve from [Stuart and Binzel, 2004] also indicates that there are approximately $180,000 \mathrm{NEAs}$ that are $\geq 100 \mathrm{~m}$ wide, indicating that surveillance efforts have discovered $<\mathbf{5 \%}$ of NEAs that are $\geq 100 \mathrm{~m}$ wide. Impact of a $100 \mathrm{~m}$ asteroid would cause severe disruption to society. For example, a $\sim 60 \mathrm{~m}$ object that entered the atmosphere and exploded over Tunguska, Russia on June 30, 1908 flattened more than $2,000 \mathrm{~km}^{2}$ of remote forest. For comparison, the city of Los Angeles, with a population of 4 million people, covers approximately $1,215 \mathrm{~km}^{2}$.

Observations from independent observatories are collected and combined into accessible databases, such as the database compiled by the Minor Planet Center (http://www.minorplanetcenter.org/). Using combined observations from data centers, orbits are determined by automated routines. NASA developed the Sentry automatic impact monitoring system for "continually updating the orbits, future close Earth approaches, and Earth impact probabilities for all NearEarth Asteroids (NEAs)" [Chamberlain et al., 2001]. The European Space Agency operates a comparable system, the Near Earth Objects Dynamic Site (NEODyS-2) [Chesley et al., 2011], using data and methods similar to Sentry.

Orbit determination allows estimation of the risk of Earth impact. Based on continuing observations, NASA's Sentry system provides daily updates of impact risks for all known objects. A list of the top 10 objects that are most likely to impact Earth is shown in Table 2 [http://neo.jpl.nasa.gov/risk/]. Asteroid $2010 \mathrm{RF} 12$, a $7 \mathrm{~m}$ wide bolide, has the highest current probability of striking Earth, with a cumulative impact probability of $8.1 \cdot 10^{-2}$ over 40 predicted close approaches spanning the years 2095 through 2112 The top-10 list contains two 130m asteroids, capable of leveling any large city, and one $560 \mathrm{~m}$ asteroid that could threaten all of humanity.

Table 2. The top 10 most probable impact threats among currently known asteroids [http://neo.jpl.nasa.gov/risk/]. One $560 \mathrm{~m}$ object and two $130 \mathrm{~m}$ objects appear in the top-10 list.

\begin{tabular}{|l|c|c|c|r|r|}
\hline Object Designation & Year Range & $\begin{array}{c}\text { Potential } \\
\text { Impacts }\end{array}$ & $\begin{array}{c}\text { Cum. Impact } \\
\text { Probability }\end{array}$ & $\begin{array}{c}\text { Closing } \\
\text { Velocity } \\
(\mathrm{km} / \mathrm{s})\end{array}$ & $\begin{array}{c}\text { Estimated } \\
\text { Diameter } \\
(\mathrm{m})\end{array}$ \\
\hline 2010 RF12 & $2095-2112$ & 40 & $8.10 \mathrm{E}-02$ & 5.10 & 7 \\
\hline 2006 JY26 & $2073-2112$ & 57 & $8.40 \mathrm{E}-03$ & 2.98 & 7 \\
\hline 2000 SG344 & $2069-2113$ & 97 & $2.40 \mathrm{E}-03$ & 1.37 & 37 \\
\hline 2009 FD & $2185-2190$ & 2 & $1.90 \mathrm{E}-03$ & 15.87 & 130 \\
\hline 2011 AM37 & $2044-2113$ & 122 & $1.10 \mathrm{E}-03$ & 3.66 & 4 \\
\hline 2009 BE & $2061-2112$ & 30 & $9.00 \mathrm{E}-04$ & 11.46 & 20 \\
\hline 2000 LG6 & $2075-2113$ & 38 & $8.90 \mathrm{E}-04$ & 2.11 & 5 \\
\hline 101955 1999 RQ36 & $2169-2199$ & 8 & $7.10 \mathrm{E}-04$ & 6.36 & 560 \\
\hline 2007 VK184 & $2048-2057$ & 4 & $5.70 \mathrm{E}-04$ & 15.63 & 130 \\
\hline 2011 BL45 & $2050-2113$ & 51 & $4.60 \mathrm{E}-04$ & 1.68 & 13 \\
\hline
\end{tabular}

\subsection{Impacts Across the Solar System}

Evidence of asteroid impacts is clearly observable throughout the solar system. Visible surfaces of rocky planets and large asteroids are all pocked with impact craters. The surface of Earth's Moon is saturated with impact craters, even though some re-shaping of the lunar surface has occurred since the moon formed. The Earth has also been subjected to a similar flux of impactors as the Moon, with several important differences. The Earth is shrouded in a dense atmosphere, which acts as a barrier to smaller impactors. Objects that penetrate the atmosphere have a greater chance of landing in an ocean than on land, and lasting craters may not form for most ocean impacts. The Earth is also geologically active. Plate tectonics continually destroys older oceanic crust, erasing any evidence of impacts that might have occurred in those areas. Uplift and erosion of continental crust continually alters impact structures, disguising or erasing most of them. At the current time, there are 184 confirmed impact structures on Earth, and several hundred additional sites designated with varying degrees of uncertainty as potential impact structures.

Perhaps the most famous confirmed impact site on Earth is the Chicxulub crater, near the Yucatán peninsula in México. The crater in Yucatán is more than $180 \mathrm{~km}$ in diameter, and represents one of the largest confirmed impact structures on Earth. An asteroid $\sim 10 \mathrm{~km}$ wide struck the Earth approximately $66.0 \mathrm{Ma}$ ago, marking the CretaceousPaleogene (K-Pg) boundary [Renne et al., 2013]. The asteroid impact is widely believed to have resulted in the extinction of non-avian dinosaurs. Recent studies have proposed that the Chicxulub crater may be related to several other impact sites, including Silver Pit in the North Sea, Boltysh in the Ukraine, and Shiva Crater in India [Chatterjee, 
1997; Keller, 2003]. Some evidence suggests that all four structures were formed at the same time, in a sequence of impacts known as a crater chain. Crater chains are commonly observed features in the solar system. Jupiter's moons Ganymede and Callisto have confirmed crater chains [Melosh and Schenk, 1993]. Potential chains have been observed on Mars [Wyrick et al, 2004], and Mercury [Watters et al., 2009]. Earth's Moon has many confirmed chains [Wilhelms and McCauley, 1971], and the Earth itself likely contains several visible chains. Eight putative impact sites ranging in size from 3 to $17 \mathrm{~km}$ wide are visible over a $700 \mathrm{~km}$ line through Kansas, Missouri, and Illinois [Rampino and Volk, 1996]. Some of the structures may be volcanic in origin, and of different ages, thus not part of the postulated 8-crater chain; however, two of the eight sites are confirmed impact structures that are both approximately $\sim 300 \mathrm{Ma}$ old, supporting the hypothesis that a crater chain of at least two impacts does exist in that area.

\subsection{Crater Chains, Rubble Piles and Keyholes}

Crater chains are thought to form when a parent bolide breaks up into multiple fragments before impacting the surface of a planet or moon [Bottke et al., 1997]. While some asteroids are clearly solid objects, a certain fraction of asteroids may be a conglomeration of many smaller fragments held together by weak gravitational force of the fragments, so-called "rubble pile" asteroids. The asteroid Itokawa, a $\sim 535 \mathrm{~m}$ wide object, is thought to be a rubble pile [Fujiwara et al., 2006]. Other studies suggest that rubble pile asteroids can be broken apart during close planetary approaches by tidal distortion [Richardson et al., 1998].

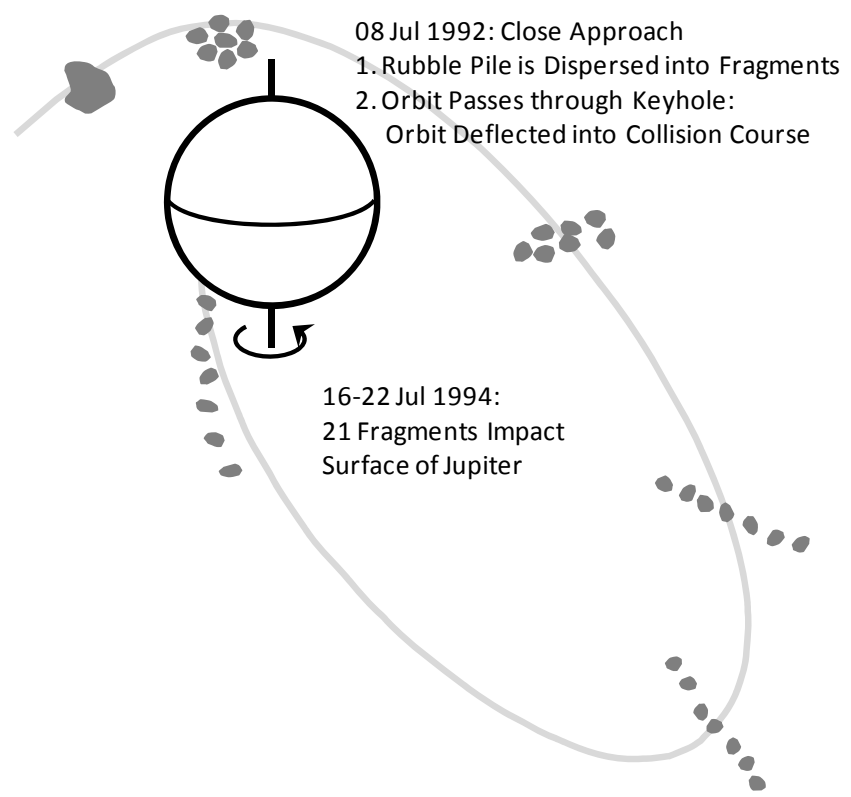

Figure 3. Final orbit of comet Shoemaker-Levy 9. At the point of close approach on July 08, 1992, twenty-one fragments ranging in size from $200 \mathrm{~m}$ to $2 \mathrm{~km}$ wide were dispersed from a parent object estimated to be in the range of 4$5 \mathrm{~km}$ wide. The close approach also passed through a keyhole, placing the final orbit on a collision course with Jupiter. The 21 fragments impacted the surface of Jupiter in a chain over a span of six days from July 16 to 22, as the planet rotated beneath the impact zone.

Formation of a crater chain was observed in great detail during the final orbit of comet Shoemaker-Levy 9 around Jupiter. Prior to a close approach to the planet on July 08, 1992, the comet was presumed to be a single 4-5km object. After the close approach, the comet broke into at least 21 separate fragments, ranging in size from $100 \mathrm{~m}$ to $2 \mathrm{~km}$. Additionally, the asteroid's orbit was altered by Jupiter's gravity in such a way as to put the comet fragments on a collision course with the planet. The comet passed through a "keyhole" during its close approach to the planet, a scenario whereby an object that had no prior chance of impacting a planet is deflected into a collision course. The 21 fragments of comet Shoemaker-Levy 9 slammed into the surface of Jupiter one after another from 16 to 22 July 1994, leaving a chain of scars in the planet's upper atmosphere as the planet rotated below the impact zones; see Fig. 3. 
The asteroid 99942 Apophis, which is $\sim 325 \mathrm{~m}$ wide and may also be a rubble pile, passed within 0.1AU of Earth on January 9, 2013; the orbit at close approach is shown in Fig. 4, taken from NASA's Near-Earth Object Program site at [http://ssd.jpl.nasa.gov/sbdb.cgi?sstr=99942;orb=1]. Predictions indicate that Apophis will have two more close approaches to Earth, one in 2029 and one in 2036. Based on current orbital information, the impact probability for both close approaches is low. However, efforts are being made to determine if Apophis will pass through a keyhole during its 2029 close approach that will put it on a collision course for the 2036 pass. If Apophis is a rubble pile, then a breakup may also be possible during the close approach in 2029, evoking thoughts of Shoemaker-Levy 9.

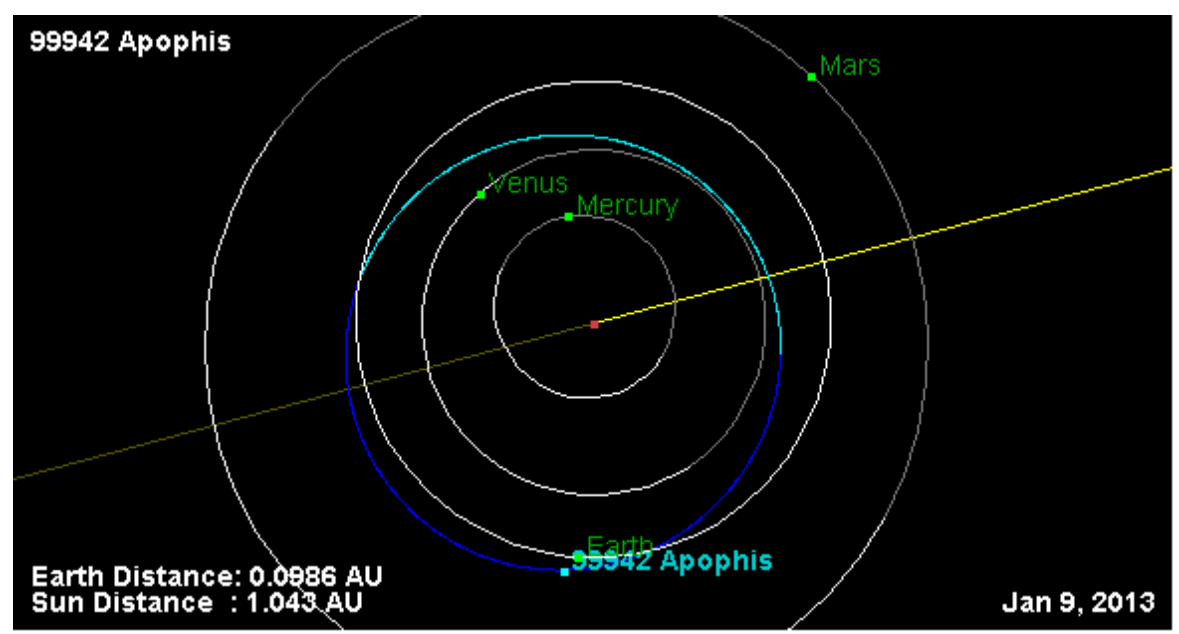

Figure 4. Orbit of 99942 Apophis. The $\sim 325 \mathrm{~m}$ bolide passed within 0.1AU of Earth on January 9, 2013. The asteroid will again pass close to Earth in 2029 and 2036. Will the close approach in 2029 alter the orbit by an amount that will put the asteroid on a collision course for 2036? Will the close approach in 2029 disperse fragments from a rubble pile core?

\section{IMPACT THREAT MITIGATION STRATEGIES}

\subsection{Survey of Current Strategies for Deflecting Threatening Objects}

Modern technological advances have enabled contemplation of methods for actively countering the threat of asteroid impact. The first published writings on the subject appear to be Project Icarus, stemming from an assignment that Professor Paul Sandorff posed to a group of MIT graduate students in 1967, seeking methods to deflect the Apolloclass asteroid 1566 Icarus [Kleiman, 1968]. The student team suggested sending six Saturn V rockets to Icarus, each armed with a 100-megaton hydrogen bomb to either deflect the asteroid's orbit, or break it up into smaller debris that would vaporize in the atmosphere. Since that time, numerous strategies for impact threat mitigation have been conceived. Several excellent surveys of impact mitigation strategies are available, including [Belton et al. (eds.), 2004], [Gritzner and Kahle, 2004], [Colombo et al., 2009], [Sanchez Cuartielles et al., 2007], and [Morrison et al., 2002].

A broad generalization of impact risk mitigation strategies divides published methods into five groups. (1) Explosive and/or kinetic impactors for orbit alteration and/or object disintegration [e.g., Koenig and Chyba, 2007; Melosh and Ryan, 1997; McInnes, 2004; Conway, 2004; many others]. (2) gradual orbit deflection with albedo alteration via paint [Hyland et al., 2010], mirrors [Vasile and Maddock, 2010], sails [Wie, 2007], etc. (3) direct motive force, such as by mounting a propellant-driven or nuclear-powered engine directly to the asteroid [Walker et al., 2005]; (4) indirect force, such as gravitational tug [Schweickart et al., 2006; Lu and Love, 2005]; (5) Vaporizing surface material with solar concentrators or lasers to create a reactionary force for orbit deflection [Gibbings et al., 2011; Maddock et al., 2007]. At least one study ([Kahle et al., 2006]) envisioned a single large reflector mounted on a spacecraft traveling alongside an asteroid; the idea was expanded to a formation of spacecraft orbiting in the vicinity of the asteroid, each equipped with a smaller concentrator assembly capable of focusing solar power onto an asteroid at distances near $\sim 1 \mathrm{~km}$ [Maddock et al., 2007; Vasile and Maddock, 2012]. 


\subsection{Stand-On Systems: Dedicated Missions for Every Individual Threat}

In studies published to date, a common feature of the tactics for impact prevention is mission emphasis on a single threatening object. Threat-specific mission strategies might be characterized as "Stand-On" approaches, since assets required for orbit alteration would need to be deployed onto, or at least very near, the threatening asteroid. Furthermore, the schemes described in the literature thus far are all complex, so successful engagement would require sufficient advance planning to design, launch and execute an intercept mission. No opportunity would exist for dry-run or experimental validation and testing, so contingency plans may require backup missions. Since $<5 \%$ of the NEA population $\geq 100 \mathrm{~m}$ wide are currently known, we should expect that multiple threats will be identified in the future by increasingly sensitive surveillance efforts; mission costs for an individual deflection mission would be large, and costs would be repeated for every threat requiring diversion. Mission costs would also be dedicated solely to impact avoidance, since equipping an orbit deflection mission with additional science capabilities may compromise the main goal. Stand-On approaches may not be practical for threats that are discovered on short notice, particularly smaller objects that may not be expected to cause extensive damage (2012 DA14?). Addressing concurrent threats, such as a Shoemaker-Levy 9 scenario on Earth (Apophis?), would add significant cost and complexity to single-target approaches.

\section{DE-STAR CONCEPT FOR ASTEROID IMPACT THREAT MITIGATION}

\subsection{DE-STAR System Concept}

We introduce DE-STAR as an alternative approach to asteroid impact risk mitigation. DE-STAR is an orbiting platform with a modular array of phase-locked laser amplifiers that is powered by solar photovoltaic panels, as depicted in Fig. 5. DE-STAR would transmit directed energy onto the surface of a distant asteroid sufficient to vaporize the surface, creating a reaction thrust that would deflect the asteroid's orbit.

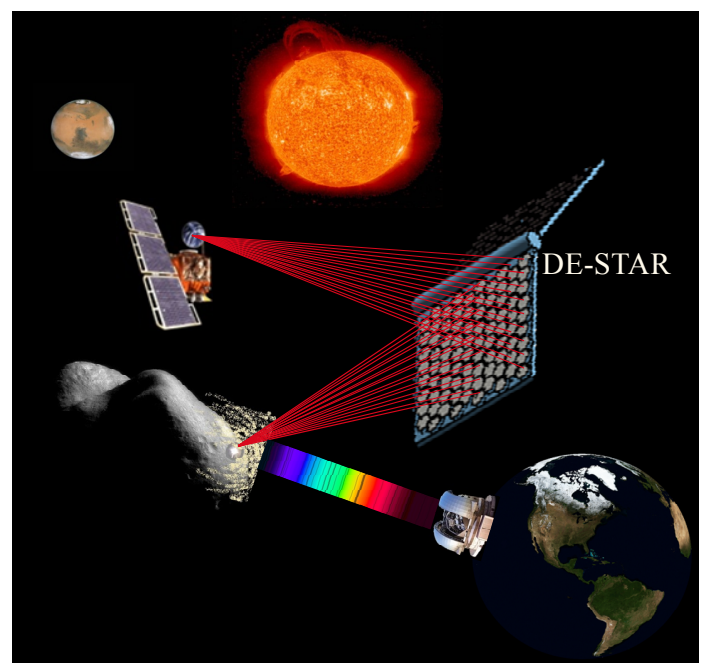

Figure 5. DE-STAR system concept for asteroid orbit deflection, and other scientific purposes. DE-STAR is an orbiting platform consisting of a solar PV panel, and a phased array of fiber laser amplifiers. Reaction to ejected material provides a thrust that deflects the asteroid's orbit. An orbiting system could also be used for other scientific purposes, such as powering interplanetary spacecraft, asteroid composition interrogation, and space debris de-orbiting.

Phased array design allows fine pointing of laser beams for delivering concentrated photon flux on the surface of an asteroid over large distances. We envision a modular approach to system development (described below), where smaller prototypes are developed in the laboratory and progressively larger systems are tested at each stage of development leading eventually to a functional, orbiting system. Laboratory devices implementing laser phased array technology exist today [Voronstov et al., 2009], and technology for scaling to larger arrays is also currently available; concept for system architecture is described below. An orbiting DE-STAR of adequate size would have the capability of reacting to small- or moderately-sized objects that are discovered with limited time to react. Additionally, a single 
orbiting DE-STAR of sufficient size could be designed to simultaneously engage multiple approaching objects. Possessing the capability to vaporize the surface of distant asteroids would also render DE-STAR capable of meeting other scientific objectives. For example, the heated spot on an asteroid's surface would emit blackbody radiation that would pass through the plume of evaporated material; viewing absorption lines with Earth-based telescopes could provide a method for determining the asteroid's composition. The directed energy spot from a DE-STAR could also be used to transmit power to other spacecraft over long distances, for example as a propulsion power source. Details of DESTAR ancillary functions are discussed in other SPIE Optics \& Photonics 2013 Proceedings papers, including [Lubin et al., 2013] and [Bible et al., 2013].

\subsection{Stand-Off Approach for Efficient and Cost-Effective Impact Risk Mitigation}

We suggest there is a fundamental difference between DE-STAR and previously described approaches to orbit deflection. All currently described concepts are "Stand-On" systems, in that assets required for orbit alteration would need to be deployed onto, or at least very near, the threatening asteroid. DE-STAR is a stand-off system that would be capable of altering an asteroid's orbit from afar. Costs associated with DE-STAR development would be amortized over multiple threats, since a functioning system could be used repeatedly. Asteroid 2012 DA14 ( 45m) was discovered one year before its close approach; could (or would?) a kinetic impact mission have been attempted, had the asteroid been on a collision course? If the object that struck near Chelyabinsk had been discovered one year (or one day) before impact, could (or would?) any stand-on mission be deployed to nullify the threat? A single DE-STAR system of modest size and flexibility would have been capable of eliminating the threats from both 2012 DA14 and the Chelyabinsk impactor (assuming prior detection by surveillance efforts). In particular, a functioning DE-STAR would be capable of mounting very rapid responses to newly-discovered objects that have no chance of being mitigated by stand-on systems. As previously stated, a single orbiting DE-STAR of sufficient size could be designed to simultaneously engage multiple approaching objects. Unlike stand-on approaches, DE-STAR could be tested and validated at every stage of development, considerably increasing confidence that the system would succeed when needed the most. Since DESTAR would be capable of addressing other scientific goals, development costs could also be spread across multiple scientific budgets.

The stand-off strategy of DE-STAR has many obvious and critical advantages over stand-on schemes currently being considered for asteroid impact avoidance. It is therefore likely worth the effort to explore the many issues associated with designing, developing and deploying an orbiting DE-STAR. In this paper, we consider baseline system requirements and architecture; cost-benefit analysis will be addressed in future work.

\section{DE-STAR SYSTEM REQUIREMENTS AND ARCHITECTURE}

\subsection{Requirements for Asteroid Deflection by Surface Vaporization}

What does it take to melt the surface of an asteroid? Energy flux requirements depend primarily on material properties. Composition of asteroids is an active research area, with recent advances coming from remote sensing spectroscopic analysis. For initial laboratory experiments, we use properties of meteorites as a first-order approximation of the range of asteroid compositions. Iron Meteorites ( $4.6 \%$ of all meteorites) are typically $25 \%$ Nickel, $75 \%$ Iron alloy, as in Antitaenite $\mathrm{Fe}_{3} \mathrm{Ni}$; the range of compositions runs from 5\% to $62 \%$ nickel with the balance of iron, as in Awaruite $\mathrm{Ni}_{2} \mathrm{Fe}$ to $\mathrm{Ni}_{3} \mathrm{Fe}$ or Kamacite $\mathrm{Fe}_{0.9} \mathrm{Ni}_{0.1}$, with some trace elements including gallium, germanium, gold, and other metals. Stony-Iron Meteorites: (1\% of all meteorites) are comprised of 30\% to $70 \%$ of weight as iron-nickel alloy as in iron meteorites, but with significant silicate rocks, including Olivine $(\mathrm{Mg}, \mathrm{Fe})_{2} \mathrm{SiO}_{4}, \mathrm{Plagioclase}_{\mathrm{KAlSi}} \mathrm{O}_{8}, \mathrm{NaAlSi}_{3} \mathrm{O}_{8}$, or $\mathrm{CaAl}_{2} \mathrm{Si}_{2} \mathrm{O}_{8}$ and Pyroxene $(\mathrm{Ca}, \mathrm{Na})(\mathrm{Mg}, \mathrm{Fe}, \mathrm{Al}, \mathrm{Ti})(\mathrm{Si}, \mathrm{Al})_{2} \mathrm{O}_{6}$. Stony Meteorites are sub-classified into several groups. Achondrites $(8.2 \%$ of all meteorites) are predominantly comprised of Olivine, Pyroxene and Plagioclase. Ordinary Chondrites $\left(80 \%\right.$ of all meteorites) contain Olivine along with Phyllosilicates such as Serpentine $\mathrm{Mg}_{3} \mathrm{Si}_{2} \mathrm{O}_{5}(\mathrm{OH})_{4}$. Carbonaceous Chondrites (4.4\% of all meteorites) are made up of Silicates $\mathrm{SiO}_{2}$, oxides of Aluminum and Iron such as $\mathrm{Al}_{2} \mathrm{O}_{3}, \mathrm{Fe}_{2} \mathrm{O}_{3}, \mathrm{Fe}_{3} \mathrm{O}_{4}$, and sulfides such as $\mathrm{FeS}_{2}, \mathrm{ZnS}$. Enstatite Chondrites (1.6\% of all meteorites) contain primarily Enstatite $\mathrm{MgSiO}_{3}$. A small fraction of meteorites $(0.2 \%)$ do not fit neatly into any of the previous categories.

Proceeding with the list of meteorite constituents, vapor pressures for common constituents can be determined experimentally, such as for Olivine [Nagahara et al., 1994]. The chart in Fig. 6 displays energy required to melt and vaporize selected substances by volume. 


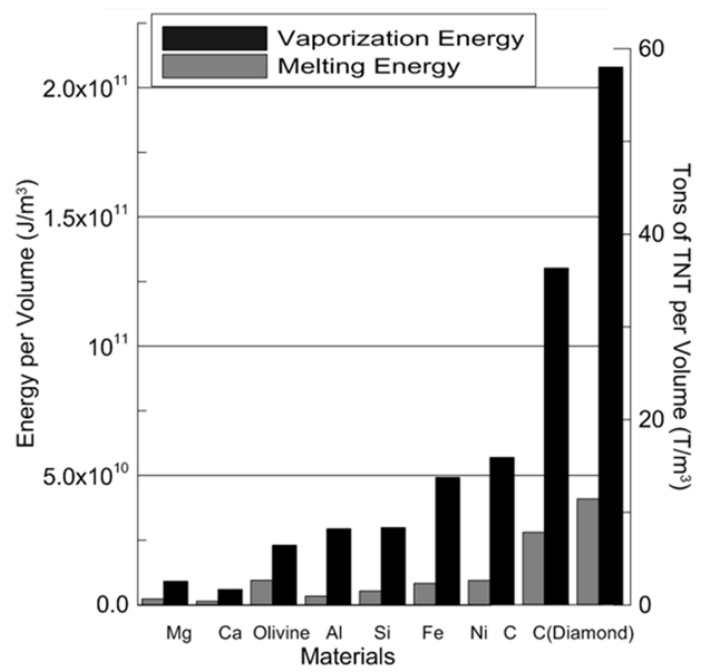

Figure 6. Energies required to melt and vaporize selected materials. Olivine is a common constituent of Ordinary Chondrites, which comprise $80 \%$ of all meteorites.

A DE-STAR system must be capable of delivering sufficient energy to vaporize materials present in asteroids. Energy transfer to the surface of an asteroid depends on several factors, including asteroid composition, surface albedo of solid and molten materials, and thermal inertia of the bolide structure. We investigated the heating profile of a hypothetical asteroid being bombarded by a directed energy beam. A dynamic finite element physical/thermal model was implemented in COMSOL, with realistic conditions for radiation, conduction and mass ejection. Model results and a simulated thermal profile are shown in Fig. 7. In the model, energy input to a heated spot was $\sim 30 \mathrm{MW} / \mathrm{m}^{2}$, which is commensurate with flux achieved using our laboratory test system (see Fig. 8). For comparison, a blackbody source at $5,700 \mathrm{~K}$, similar to the Sun's surface, generates approximately $60 \mathrm{MW} / \mathrm{m}^{2}$, and is far more than sufficient to vaporize any material that is subjected to such a flux.
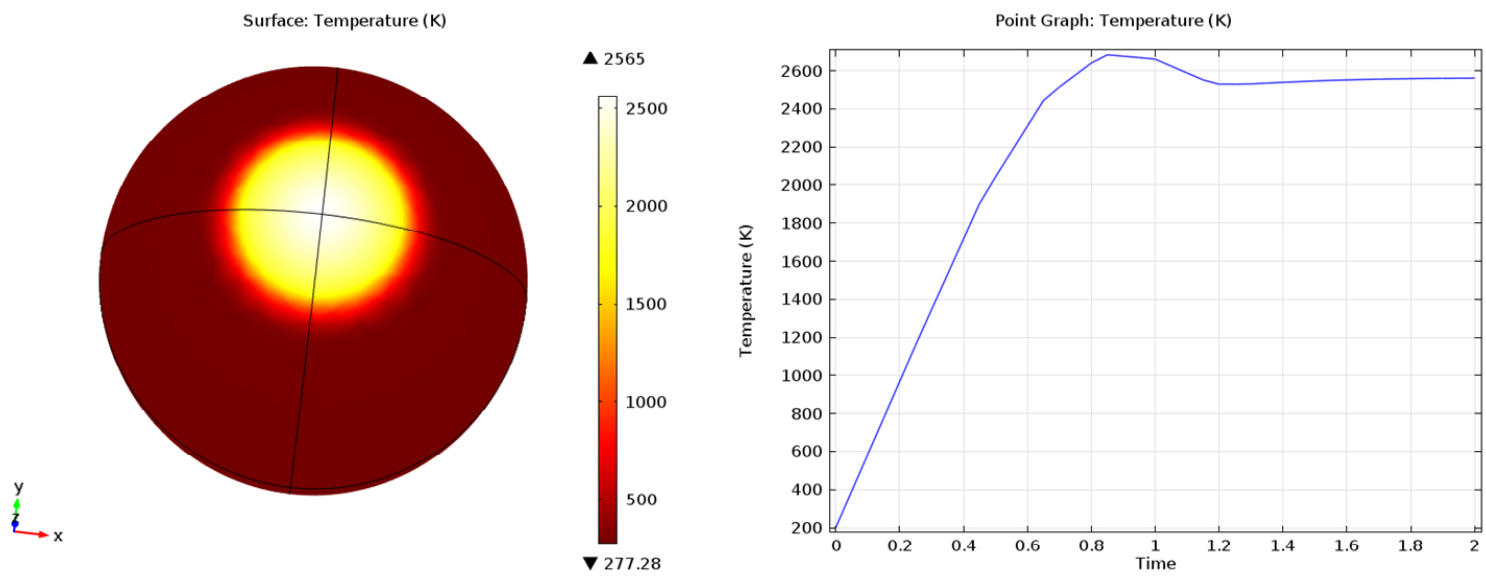

Figure 7. COMSOL model for a spherical bolide of micro-crystalline Silica $\left(\mathrm{SiO}_{2}\right)$. Model features include radiation, conduction and mass ejection. Left figure is steady-state condition. Right figure is initial thermal profile at the central spot; time axis is in seconds. The model suggests that vaporization commences within 1 second; the thermal profile stabilizes at a level consistent with the vaporization temperature of Silica in vacuum.

To investigate applicability of the physical/thermal models to real-world materials, a laboratory test system was developed, as shown in Fig. 8 and the accompanying video. An array of 19 fiber laser with a total power of 40 watts was mounted approximately $10 \mathrm{~cm}$ away from target materials. The laser energy was focused in a circular spot $\sim 1 \mathrm{~mm}$ in 
diameter, producing a flux of $>10 \mathrm{MW} / \mathrm{m}^{2}$. Samples of various materials, including Basalt, Pumice, sandstone, beach sand (and milk chocolate) were tested as targets. All of the tested materials were vaporized. Additionally, thermal profiles of selected tests were recorded with a thermally-calibrated long-wave infrared camera, at 30 frames per second. Experimental results confirm that vaporization is initiated within $1 \mathrm{sec}$. In the accompanying video, copious amounts of ejected material are clearly evident in the infrared sequence.

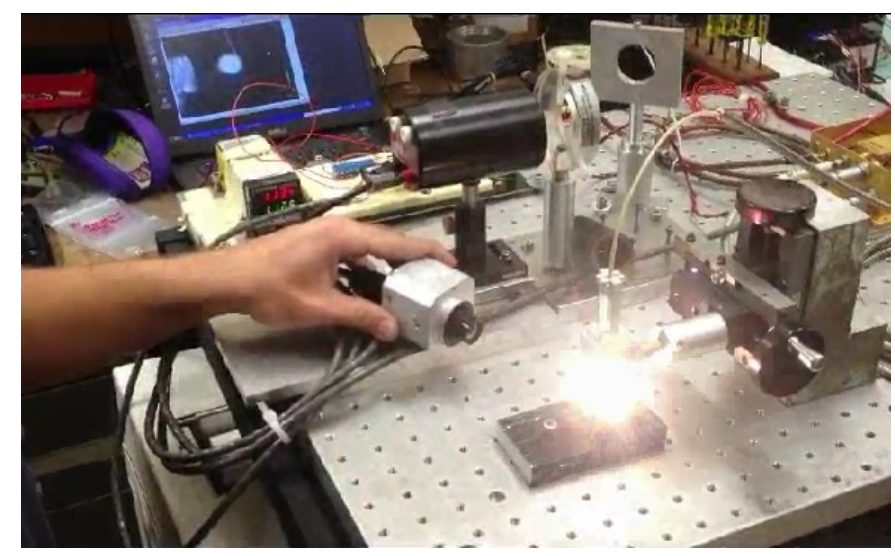

Figure 8/Video 1: http://dx.doi.org/10.1117/12.2026401.1 Laboratory test systemto check predictions of thermal modeling. A multi-element fiber laser is focused through a lens on a targets (in this picture it is largely $\mathrm{SiO}_{2}$ from local sand). The heated spot is viewed with a thermal infrared camera, and time evolution of the temperature distribution of the basalt surface is recorded in the infrared video.

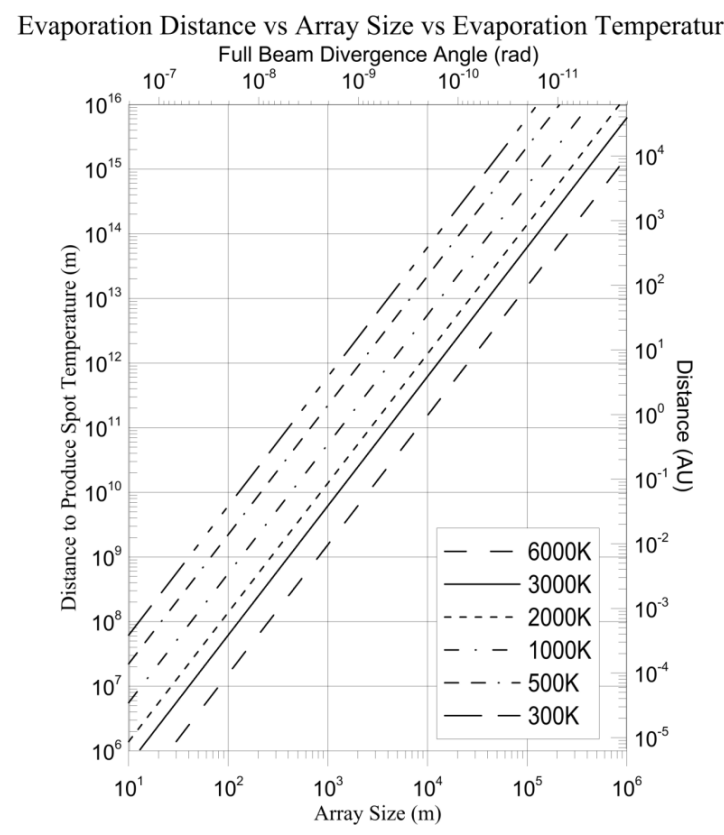

Figure 9. Various materials present in asteroids will melt and vaporize at different temperatures. The distance at which evaporation can occur is plotted against array size for substances with selected melting temperatures.

We use power flux requirements for surface evaporation gleaned from the thermal model and experimental results to estimate laser phased array sizes that would be required to achieve selected temperature values over a range of target distances; results are presented in Fig. 9. For a surface temperature at the vaporization point of Silica in vacuum $(\sim 2,600 \mathrm{~K})$, and a target at $0.01 \mathrm{AU}$, an array of $\sim 250 \mathrm{~m}$ by $\sim 250 \mathrm{~m}$ would be required. Slower volatile-laden objects 
$\sim 100 \mathrm{~m}$ wide and approaching at $5 \mathrm{~km} / \mathrm{s}$ could be diverted by initiating engagement at $\sim 0.05 \mathrm{AU}$, requiring a laser array of $\sim 100 \mathrm{~m}$ side length [Lubin et al., 2013]. Vaporization of space debris in Earth orbit could be accomplished with a $\sim 10 \mathrm{~m}$ array [Lubin et al., 2013]. Full planetary defense against all known threats requires between a $10^{3}$ and $10^{4} \mathrm{~m}$ array.

\subsection{Phased Array Laser Architecture}

The system architecture for a phased-array laser consists of a seed laser (analogous to a master oscillator in a phased-array radar system); the seed output is routed at low intensity to each laser sub-element. Each sub-element has a fiber laser amplifier for the seed laser signal (analogous to electronic amplifiers in a phased-array radar system). Exit wavefront relationships are fed back to control the output phase of each fiber amplifier. Individual beam phases are adjusted precisely to phase-lock each fiber amplifier in relation to the seed laser and feedback signal. By controlling the phase output of each fiber amplifier, the combined beam from the array of sub-elements can be steered precisely (similar principles as a phased-array radar system). In larger laser phased array systems, the beams do not comingle and combine near the source, but rather near the target, so the system is described as a Synthetic Aperture Laser Phased Array (SALPA). To accomplish beam combination near the target in a SALPA configuration, fiber tip displacements to appropriate off-axis positions behind each transmitter provide coarse orientation of the beam toward the target. Fine beam steering and combining is then accomplished by phase control. This scheme requires wavefront sensing at each transmitter exit, and precision structural metrology of the fiber array transmitter. Laboratory systems with the required characteristics have been developed by [Voronstov et al., 2009], depicted in Fig. 10, using Ytterbium (Yb) fiber laser amplifiers.

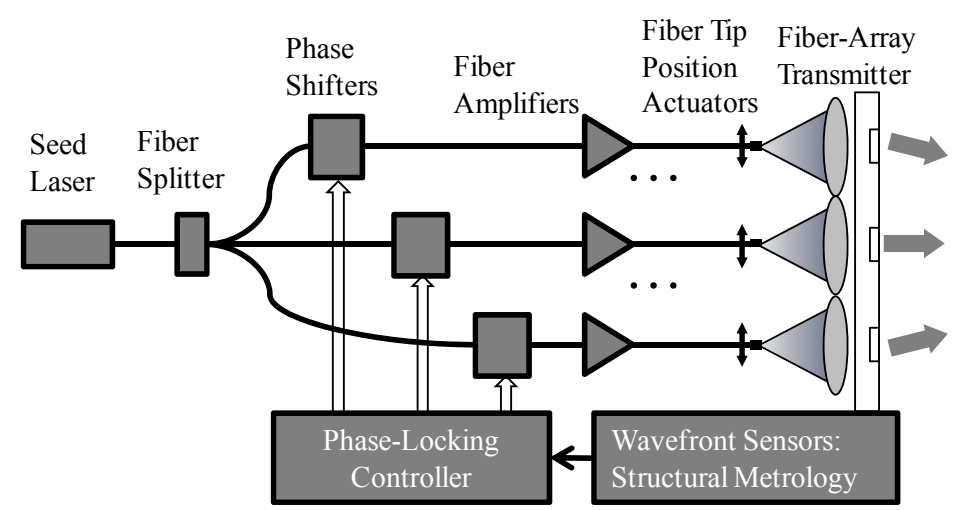

Figure 10. A system block diagram of the fiber amplifier configuration, based on work by [Voronstov et al., 2009]. Individual beams combine near the target. Coarse beam orientation is accomplished by moving individual fiber amplifier tips in relation to the transmitting element. Fine beam steering and beam combination at the target is accomplished by phase control.
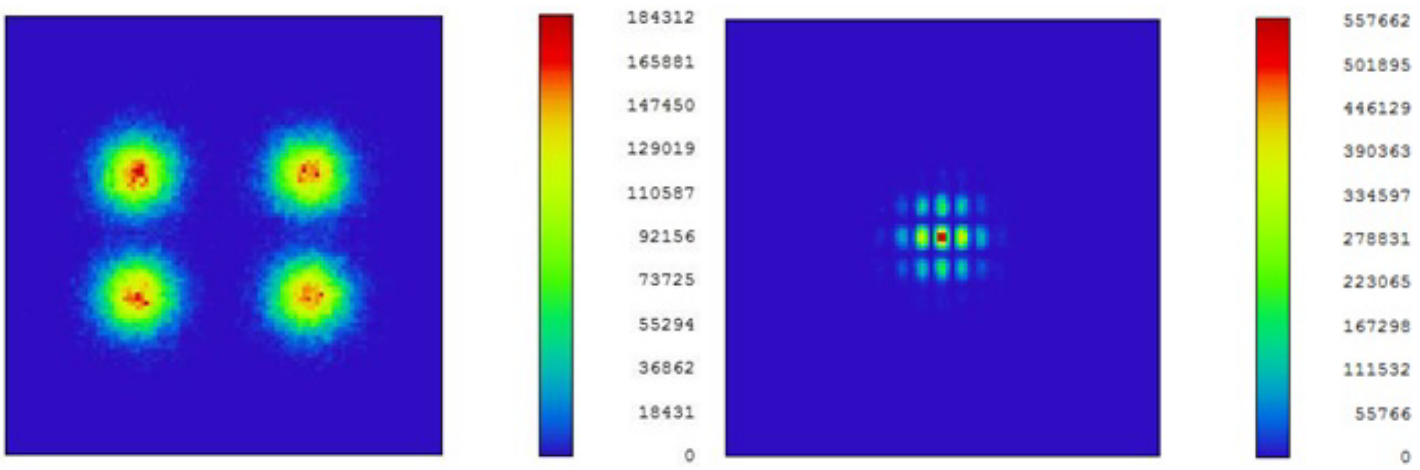

Figure 11. Zemax model of a 4-element laser phased array. Left panel shows un-combined beam power distribution, with peak flux in the range of $1.8 \mathrm{MW} / \mathrm{m}^{2}$ (actual units are arbitrary). As the phase of each individual beam is controlled, a central combined lobe forms with peak flux in the range of $5.6 \mathrm{MW} / \mathrm{m}^{2}$, clearly showing combination of power from individual beams in the central lobe. As more elements are added the peak combined flux at a given target distance grows roughly as $\mathrm{N}^{2}$ where $\mathrm{N}$ is the number of elements. This assumes a relatively dense packed array with identical elements. 
Optical modeling of a 4-element system based on the design shown in Fig. 9 was demonstrated in Zemax; results are shown in Fig. 11. In the left panel of Fig. 11, the individual un-combined beam patterns are displayed. When beam orientation and phase control are introduced, the beams combine into the pattern shown in the right panel of Fig. 11. Side-lobe power is present in the combined beam pattern, but combination of power from the individual signals into the central lobe is clearly evident. As array size increases, side-lobe power will still be present, but the central combined beam will be capable of meeting requirements for vaporization of substances present in asteroids, as determined by the physical/thermal model shown in Fig. 7 and experimental results depicted in Fig. 8.

\subsection{Solar Photovoltaic Panels for Power}

Our vision for DE-STAR is that power for the laser phased array would be supplied by solar photovoltaic (PV) panels. Current laser fiber amplifier technology is capable of generating $1 \mathrm{~kW}$ of laser power with $\sim 5 \mathrm{~kg}$ of apparatus, with over $35 \%$ conversion efficiency of electricity to laser power. Heat dissipation requirements of the laser apparatus indicate that a reasonable target for power density in the laser phased array is approximately $1 \mathrm{~kW} / \mathrm{m}^{2}$. Space PV efficiency is now approaching 50\%, for example Soitec's 4-junction PV cell [http://www.solarreviews.com/blog/SoitecDebuts-4-Junction-PV-Cell-5-28-13/]. The solar constant at the top of Earth's atmosphere is the amount of energy flux from the sun reaching the outer atmosphere; the value varies from $1.361 \mathrm{~kW} / \mathrm{m}^{2}$ in early July to $1.412 \mathrm{~kW} / \mathrm{m}^{2}$ in early January as the distance from Earth to the Sun varies (values given are for solar cycle minimum; at solar cycle maximum, the values are $\sim 0.1 \%$ higher). Combined efficiency of the fiber amplifiers and solar cells at current conversion efficiencies results in $\sim 0.25 \mathrm{~kW}$ of laser output per square meter of solar PV. Alternatively, to produce $1 \mathrm{~kW}$ of laser output, $\sim 4 \mathrm{~m}^{2}$ of solar PV is required, at current conversion efficiencies. It is not unreasonable to anticipate that conversion efficiencies of both the fiber amplifiers and solar PV cells will increase as development of a DE-STAR system proceeds. The current level of efficiency and power density is sufficient to begin building the smaller units (up to DE-STAR 2 is already feasible) and with anticipated development already underway the power density of fiber amplifiers is expected to rise by nearly an order of magnitude in the next decade and efficiency will likely rise roughly a factor of 2. The power density and efficiency of space solaris similarly expected to rise over the next decade. Currently both laser amplifiers and space PV have about the same power density $(\mathrm{kW} / \mathrm{kg})$ and efficiency.

\section{PHASED DEVELOPMENT DE-STAR}

Laser phased array systems are currently available, and devices such as those described by [Voronstov et al., 2009] have been built with more than 20 elements. Extending the laboratory technology to a large orbiting system capable of planetary defense would represent a giant leap, with significant technological hurdles. However, one of the key advantages of contemplating the use of laser phased arrays for planetary defense is that stepped-development is available, with low risk and relatively low initial cost, as well as the opportunity for validation testing at every development step. We suggest laboratory tests and sub-orbital, balloon-borne flights as preliminary steps in a roadmap for achieving a DE-STAR system capable of planetary defense and other scientific objectives.

To be effective, laser driven directed energy systems require both sufficient total power and excellent beam convergence to yield adequate flux on target. Several factors have crucial impact on beam convergence, including optical aperture size, pointing control and jitter, transmission distortion of the beam, efficacy of adaptive optics strategies and other factors. Fig. 12 depicts a conceptual scheme for testing laser phased array performance in the upper atmosphere, using balloons to deploy the directed energy system components. Existing balloon technology is capable of delivering payloads up to $165,000 \mathrm{ft}(50 \mathrm{~km})$, well into the stratosphere, where pressures are much lower than the service ceiling of aircraft. The effective range of a laser at such altitudes is far greater than ranges attainable by lasers carried on aircraft due to extremely low turbulence, low atmospheric absorption, extremely large stable optics and much greater horizon distance. A two-balloon system would deliver laser and optical payloads into the stratosphere with line-of-sight distances exceeding $870 \mathrm{mi}(1,400 \mathrm{~km})$. Optical systems on each platform would provide measurements of laser beam distortion at altitude, and onboard sensors with meter-class optics would measure flux on target. Before field testing can be contemplated, a series of experiments is required to determine system-level specifications for the scheme outlined in Fig. 12. Within the context of balloon-borne systems, aspects of system mechanical and optical design that affect beam convergence can be tested in the laboratory, with the larger objective of establishing system design specifications for a successful stratospheric, long-range directed energy system. Our group has 35 years of experience in developing and flying precision pointed balloon borne platforms that can fly above $40 \mathrm{~km}$ altitude. 
Results of sub-orbital tests could be used to inform design concepts for a small orbiting system. Launch of an operational phased array laser and PV panel would be costly, but design risks are substantially mitigated by validation during sub-orbital tests. Any orbital system would require station keeping capability, so leveraging an existing orbital platform such as the International Space Station (ISS) could also lower risks associated with development of a functional, orbiting DE-STAR.

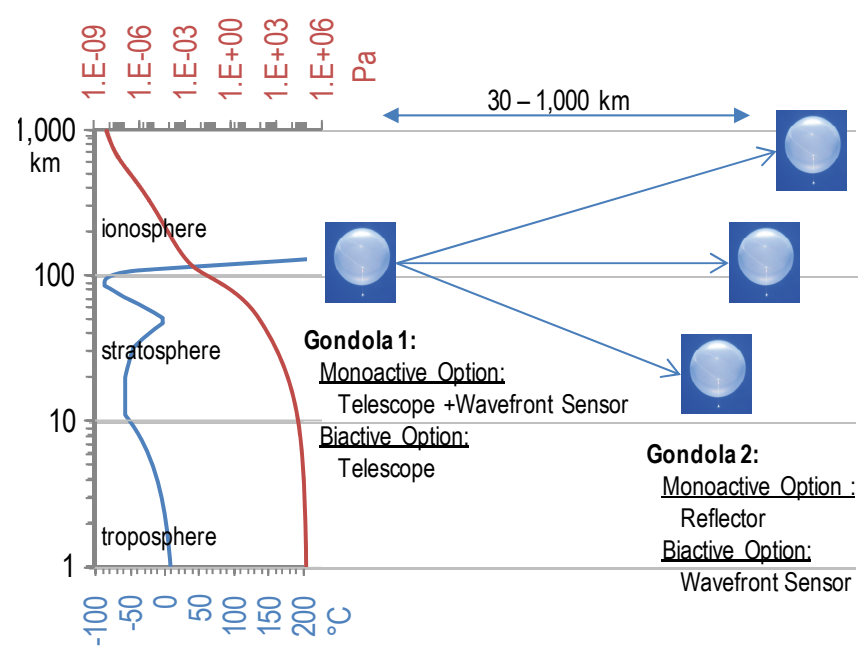

Figure 12. A long-range, two-balloon concept for measuring laser beam propagation in the upper atmosphere. The monoactive option has one active balloon (Gondola 1 hosting both telescope transmitter and wavefront sensor receiver) and a passive retro-reflector on Gondola 2, featuring double-pass propagation range. The biactive option separates telescope and sensor using two active balloons, and features single-pass propagation measurements.

\section{CONCLUSIONS}

Results of physical/thermal models, and laboratory testing, demonstrate that small-scale laser systems are capable of vaporizing materials found in asteroids over small distances. Laser phased arrays have been developed in the laboratory, and have already been scaled up to produce systems capable of vaporizing known materials over considerable distances. Continued scaling of laser phased array technology could lead to devices that would be capable of vaporizing materials over extremely large distances. We propose development of such a stand-off system, called DE-STAR, to be deployed into Earth orbit for planetary defense. A DE-STAR of sufficient size could be used to modify the orbit of asteroids, including diverting asteroids from a collision course with Earth. A functional system would possess the capability of reacting to small- or moderately-sized objects that are discovered with limited time to react. Systems of sufficient size and flexibility could engage multiple targets simultaneously. Stepped development, beginning with existing technology and proceeding with validation at every design stage, provides a low-risk and relatively low-initialcost approach for pursuing a functioning DE-STAR. Achieving an orbital system would also serve other scientific objectives, such as interrogation of asteroid composition and as a power source for interplanetary spacecraft propulsion. A stand-off system for planetary defense possesses numerous key benefits over previously-described stand-on systems that would need to be deployed onto, or at least very near, the threatening asteroid to be effective. The evident merits of DE-STAR for planetary defense and other scientific purposes should spur serious consideration for further study and development.

\section{ACKNOWLEDGEMENTS}

We acknowledge funding from the NASA California Space Grant NASA NNX10AT93H in support of this research. We are grateful to the Zemax support team for the optical simulations. 


\section{REFERENCES}

[1] Belton, Michael JS, Thomas H. Morgan, Nalin H. Samarasinha, and Donald K. Yeomans, eds. Mitigation of hazardous comets and asteroids. Cambridge University Press, 2004.

[2] Bible, Johanna, Isabella Johansson, Gary B. Hughes, and Philip M. Lubin. "Relativistic Propulsion Using Photonic Directed Energy." Proc. Of SPIE, Optics \& Photonics (2013).

[3] Bottke Jr, William F., Derek C. Richardson, and Stanley G. Love. "Can Tidal Disruption of Asteroids Make Crater Chains on the Earth and Moon?" Icarus 126.2 (1997): 470-474.

[4] Chamberlin, A. B., S. R. Chesley, P. W. Chodas, J. D. Giorgini, M. S. Keesey, R. N. Wimberly, and D. K. Yeomans. "Sentry: an automated close approach monitoring system for near-Earth objects." In Bulletin of the American Astronomical Society, vol. 33, p. 1116. 2001.

[5] Chatterjee, Sankar. "Multiple Impacts at the KT Boundary and the Demise of the Dinosaurs." Comparative planetology, geological education, history of geology: proceedings of the 30th International Geological Congress, Beijing, China, 4-14 August 1996. Vol. 26. CRC Press, 1997.

[6] Chesley, S, A.M. Comparetti, G.B. Valsecchi, F. Bernardi, D. Farnocchia, A. Chessa, D.B. Cioci, N. Ronci and R. Guerriero, NEODyS-2 (2011), http://newton.dm.unipi.it/neodys2/

[7] Cockell, Charles. "A Scientific Impact Response Team for the Aftermath of Small Asteroid and Comet Impacts." Science and Global Security 13, no. 1-2 (2005): 105-115.

[8] Colombo, Camilla, Massimiliano Vasile, and Gianmarco Radice. "Semi-analytical solution for the optimal low-thrust deflection of near-earth objects." Journal of Guidance, Control, and Dynamics 32.3 (2009): 796809.

[9] Conway, Bruce A. "Optimal interception and deflection of Earth-approaching asteroids using low-thrust electric propulsion." Mitigation of hazardous comets and asteroids Belton MJS, Morgan TH, Samarasinha N, Yeomans DK (2004): 292-312.

[10] Fujiwara, Akira, J. Kawaguchi, D. K. Yeomans, M. Abe, T. Mukai, T. Okada, J. Saito et al. "The rubble-pile asteroid Itokawa as observed by Hayabusa." Science 312, no. 5778 (2006): 1330-1334.

[11] Gibbings, Alison. "On Testing Laser Ablation Processes."

http://www.strath.ac.uk/media/faculties/engineering/advancedspaceconceptslab/research/optimisation/PDC_Pr esentation (laser_.pdf

[12] Gritzner, Christian, and Ralph Kahle. "Mitigation technologies and their requirements." In Mitigation of Hazardous Comets and Asteroids, vol. 1, p. 167. Cambridge Univ. Press, Cambridge (2004).

[13] Helin, Eleanor F., Steven H. Pravdo, David L. Rabinowitz, and Kenneth J. Lawrence. "Near-Earth Asteroid Tracking (NEAT) Program." Annals of the New York Academy of Sciences 822, no. 1 (1997): 6-25.

[14] Hyland, D. C., H. A. Altwaijry, S. Ge, R. Margulieux, J. Doyle, J. Sandberg, B. Young, X. Bai, J. Lopez, and N. Satak. "A permanently-acting NEA damage mitigation technique via the Yarkovsky effect." Cosmic Research 48, no. 5 (2010): 430-436.

[15] Kahle, Ralph, Gerhard Hahn, and Ekkehard Kührt. "Optimal deflection of NEOs en route of collision with the Earth." Icarus 182, no. 2 (2006): 482-488.

[16] Kaiser, Nicholas, Herve Aussel, Barry E. Burke, Hans Boesgaard, Ken Chambers, Mark R. Chun, James N. Heasley et al. "Pan-STARRS: a large synoptic survey telescope array." In Astronomical Telescopes and Instrumentation, pp. 154-164. International Society for Optics and Photonics, 2002.

[17] Keller, G., Stinnesbeck, W., Adatte, T., \& Stüben, D. (2003). Multiple impacts across the Cretaceous-Tertiary boundary. Earth-Science Reviews, 62(3), 327-363.

[18] Kleiman, Louis A., "Project Icarus: an MIT Student Project in Systems Engineering." Cambridge, Massachusetts: MIT Press, 1968.

[19] Koenig, J.D., and Chyba, C.F. "Impact deflection of potentially hazardous asteroids using current launch vehicles." Science and Global Security 15, no. 1 (2007): 57-83.

[20] Larson, Stephen M., E. C. Beshore, E. J. Christensen, R. E. Hill, R. A. Kowalski, A. R. Gibbs, A. P. Odell, R. H. McNaught, G. J. Garradd, and A. D. Grauer. "The status of the Catalina Sky Survey for NEOs." In Bulletin of the American Astronomical Society, vol. 38, p. 592. 2006.

[21] Lu, Edward T., and Stanley G. Love. "A gravitational tractor for towing asteroids." arXiv preprint astro$\mathrm{ph} / 0509595$ (2005).

[22] Lubin, Philip, Gary B. Hughes, Johanna Bible, Jesse Bublitz, Josh Arriola, Caio Motta, Jon Suen, Isabella Johansson, Jane Wu, Andrew Milich, Mitch Oleson and Mark Pryor. "DE-STAR: A Planetary Defense and Exploration System.” Proc. Of SPIE, Optics \& Photonics (2013).

[23] Maddock, Christie, Joan Pau Sanchez Cuartielles, Massimiliano Vasile, and Gianmarco Radice. "Comparison of Single and Multi-Spacecraft Configurations for NEA Deflection by Solar Sublimation." In AIP Conference Proceedings, vol. 886, p. 303. 2007. 
[24] Manley, Scott, Asteroid Discovery - 1980-2012 - UHDTV

http://www.youtube.com/watch?v=xJsUDcSc6hE\&feature $=$ c4-overviewvl\&list=PLYu7z3I8tdEn2m lLL3Vn7BDwkvMLo hl

[25] McInnes, Colin R. "Deflection of near-Earth asteroids by kinetic energy impacts from retrograde orbits." Planetary and Space Science 52.7 (2004): 587-590.

[26] Melosh, H. J., and E. V. Ryan. "Asteroids: Shattered but not dispersed." Icarus 129, no. 2 (1997): 562-564.

[27] Melosh, Henry Jay, and P. Schenk. "Split comets and the origin of crater chains on Ganymede and Callisto." (1993): 731-733.

[28] Morrison, David, Alan W. Harris, Geoffrey Sommer, Clark R. Chapman, and Andrea Carusi. "Dealing with the impact hazard." Asteroids III"(ed. W. Bottke et al., Univ. Ariz. Press) (2002): 739-754.

[29] Nagahara, Hiroko, Ikuo Kushiro, and Bjorn O. Mysen. "Evaporation of olivine: Low pressure phase relations of the olivine system and its implication for the origin of chondritic components in the solar nebula." Geochimica et cosmochimica acta 58.8 (1994): 1951-1963.

[30] M.R. Rampino and T. Volk, Multiple impact event in the Paleozoic: Collision with a string of comets or asteroids? (1996) Geophys. Res. Lett. 23, p. 49.

[31] Renne, Paul R., et al. "Time scales of critical events around the Cretaceous-Paleogene boundary." Science 339.6120 (2013): 684-687.

[32] Richardson, Derek C., William F. Bottke, and Stanley G. Love. "Tidal distortion and disruption of Earthcrossing asteroids." Icarus 134.1 (1998): 47-76.

[33] Sanchez, S., J. Nomen, M. Hurtado, J. A. Jaume, W. K. Y. Yeung, P. Rios, F. Serra, F. Serra (Observers at Observatorio Astronómico de La Sagra, Granada, Spain), Minor Planet Electronic Circulars, 2012, discovery chronicled at the Minor Planet Center web site: http://www.minorplanetcenter.net/mpec/K12/K12D51.html.

[34] Cuartielles, Joan Pau Sanchez, et al. "A Multi-criteria Assessment of Deflection Methods for Dangerous NEOs." AIP conference proceedings. Vol. 886. 2007.

[35] Schweickart, Russell, Clark Chapman, Dan Durda, and Piet Hut. "Threat mitigation: the gravity tractor." arXiv preprint physics/0608157 (2006).

[36] Stokes, Grant H., Jenifer B. Evans, Herbert EM Viggh, Frank C. Shelly, and Eric C. Pearce. "Lincoln nearEarth asteroid program (LINEAR)." Icarus 148, no. 1 (2000): 21-28.

[37] Stuart, Joseph Scott. "A near-Earth asteroid population estimate from the LINEAR survey." Science 294.5547 (2001): 1691-1693.

[38] Stuart, Joseph Scott, and Richard P. Binzel. "Bias-corrected population, size distribution, and impact hazard for the near-Earth objects." Icarus 170.2 (2004): 295-311.

[39] Vasile, Massimiliano, and Christie Alisa Maddock. "On the deflection of asteroids with mirrors." Celestial Mechanics and Dynamical Astronomy 107, no. 1 (2010): 265-284.

[40] Vorontsov, Mikhail A., et al. "Adaptive array of phase-locked fiber collimators: analysis and experimental demonstration." Selected Topics in Quantum Electronics, IEEE Journal of 15.2 (2009): 269-280.

[41] Walker, Roger, Dario Izzo, Cristina de Negueruela, Leopold Summerer, Mark Ayre, and Massimiliano Vasile. "Concepts for Near-Earth Asteroid deflection using spacecraft with advanced nuclear and solar electric propulsion systems." Journal of the British Interplanetary Society 58, no. 7-8 (2005): 268-278.

[42] Wallace, Brad, Frank Pinkney, Robert Scott, Donald Bedard, Jim Rody, Aaron Spaans, Martin Levesque et al. "The Near Earth Orbit Surveillance Satellite (NEOSSat)." In 55th International Astronautical Congress 2004, pp. 1-9. 2004.

[43] Watters, Thomas R., James W. Head, Sean C. Solomon, Mark S. Robinson, Clark R. Chapman, Brett W. Denevi, Caleb I. Fassett, Scott L. Murchie, and Robert G. Strom. "Evolution of the Rembrandt impact basin on Mercury." Science 324, no. 5927 (2009): 618-621.

[44] Wie, Bong. "Hovering control of a solar sail gravity tractor spacecraft for asteroid deflection." In Proceedings of the 17th AAS/AIAA Space Flight Mechanics Meeting, AAS, vol. 7, p. 145. 2007.

[45] Wilhelms, Don E., and John F. McCauley. Geologic map of the near side of the Moon. US Geological Survey, 1971.

[46] Wlodarczyk, I. "The potentially dangerous asteroid 2012 DA14." Monthly Notices of the Royal Astronomical Society 427.2 (2012): 1175-1181.

[47] Wyrick, Danielle, David A. Ferrill, Alan P. Morris, Shannon L. Colton, and Darrell W. Sims. "Distribution, morphology, and origins of Martian pit crater chains." Journal of geophysical research 109, no. E6 (2004): E06005. 